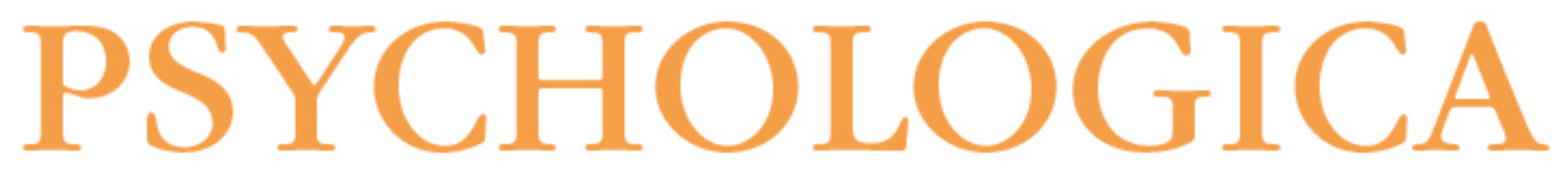

Stress parental no período pós-parto: adaptação do parental stress inventory para a população portuguesa

Autor(es): $\quad$ Gomez, Rita; Leal, Isabel

Publicado por: Imprensa da Universidade de Coimbra

URL

persistente: URI:http://hdl.handle.net/10316.2/5431

DOI: DOI:http://dx.doi.org/10.14195/1647-8606_50_19

Accessed : $\quad$ 26-Apr-2023 03:48:05

A navegação consulta e descarregamento dos títulos inseridos nas Bibliotecas Digitais UC Digitalis, UC Pombalina e UC Impactum, pressupõem a aceitação plena e sem reservas dos Termos e Condições de Uso destas Bibliotecas Digitais, disponíveis em https://digitalis.uc.pt/pt-pt/termos.

Conforme exposto nos referidos Termos e Condições de Uso, o descarregamento de títulos de acesso restrito requer uma licença válida de autorização devendo o utilizador aceder ao(s) documento(s) a partir de um endereço de IP da instituição detentora da supramencionada licença.

Ao utilizador é apenas permitido o descarregamento para uso pessoal, pelo que o emprego do(s) título(s) descarregado(s) para outro fim, designadamente comercial, carece de autorização do respetivo autor ou editor da obra.

Na medida em que todas as obras da UC Digitalis se encontram protegidas pelo Código do Direito de Autor e Direitos Conexos e demais legislação aplicável, toda a cópia, parcial ou total, deste documento, nos casos em que é legalmente admitida, deverá conter ou fazer-se acompanhar por este aviso. 


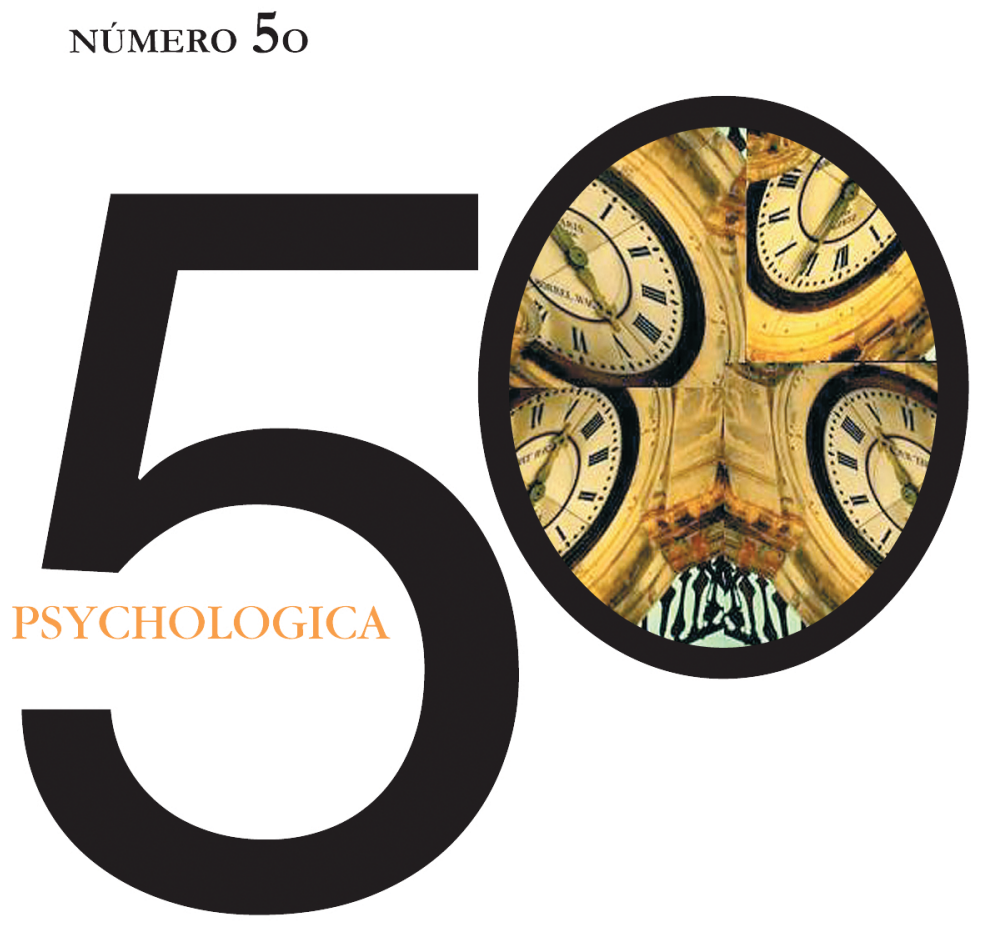

IMPRENSA DA UNIVERSIDADE DE COIMBRA

FACULDADE DE PSICOLOGIA E DE CIÊNCIAS DA EDUCAÇÃO DA UNIVERSIDADE DE COIMBRA 


\title{
Stress parental no período pós-parto: Adaptação do Parental Stress Inventory para a população portuguesa
}

Rita Gomez \& Isabel Leal'

\begin{abstract}
Neste estudo apresentam-se os resultados da adaptação para a população portuguesa do Stress Parental Inventory (PSI; D.J. Terry, 1991). No geral, os resultados indicam que a versão portuguesa do PSI (ou Inventário de Stress Parental no Pós-Parto) é uma medida fidedigna e válida para avaliar o nível de stress parental dos progenitores no período pós-parto. A escala apresenta um nível de consistência interna elevado $(\alpha=0,862)$. Verificou-se também que as notas maternas e paternas se correlacionam positivamente entre si e com medidas de ansiedade e de depressão, e negativamente com medidas de ajustamento conjugal e de envolvimento paterno.
\end{abstract}

PALAVRAS-CHAVE: Inventário de Stress Parental no Pós-Parto; Parentalidade; Gravidez; Família.

\section{Introdução}

O período da gravidez e do nascimento de um filho constitui uma fase desenvolvimental crítica que envolve vários reajustamentos a nível individual, conjugal e familiar (Colman \& Colman, 1991; Relvas \& Lourenço, 2001). Durante a gravidez, este processo adaptativo é normalmente acompanhado de ansiedade acrescida, que na maioria dos casos é de natureza transitória e de intensidade moderada e diminui com o nascimento de um bebé saudável (Dulude et al., 2002).

Contudo, a adaptação à parentalidade é influenciada por factores de variada ordem - relacionais, individuais e contextuais - e varia no tempo, de casal para casal e de indivíduo para indivíduo. Frequentemente, como referem Delmore-Ko et al. (2000), a experiência factual depois do nascimento é diferente daquilo que os progenitores esperaram e muitos dos stresses normais do período pós-parto não foram antecipados. Para as mulheres, a adaptação pode ser particularmente difícil já que são elas que tipicamente assumem a responsabilidade primária pelos 
cuidados ao bebé (Deutsch et al., 2001; Dienheart, 2001) e as suas expectativas sobre a participação paterna são muitas vezes insatisfeitas (Coffman et al., 1991; Levitt et al., 1993). De facto, embora nem todas as mulheres reportem um decréscimo da satisfação conjugal depois do nascimento e algumas reportem mesmo um aumento (Delmore-Ko et al., 2000), tem sido consistentemente demonstrado que a satisfação conjugal materna declina quando as expectativas pré-natais não são confirmadas (Levitt et al., 1994). Há também evidência de que, em termos médios, os homens reportam menor decréscimo da satisfação conjugal do que as mulheres (Levy-Shiff, 1994; Dulude et al., 2002)

Para além da vida doméstica, o nascimento de um filho implica frequentemente mudanças importantes noutras áreas, tais como finanças, actividades de lazer, relação com os familiares e amigos, e vida profissional, que afectam também os novos pais. No estudo de Ausloos (1996), todos os casais relataram ter passado por um período de crise pessoal durante os primeiros seis meses pós-parto. Levy-Shiff (1999) explorou o ajustamento dos homens à parentalidade, usando o modelo teórico do stress e coping de R.S. Lazarus (1993), e verificou que uma percentagem significativa reportava stress acrescido no fim do primeiro ano pós-parto. Muitos homens sofrem também um aumento de sintomatologia depressiva depois do nascimento. A incidência de depressão paterna pós-natal varia entre 1,2 e 25,5\% em amostras comunitárias (e.g. Areias et al., 1996; Matthey et al., 2000, 2003) e entre 24 a 50\% nos homens com companheiras deprimidas (Goodman, 2004). Nas mulheres, ainda que a sintomatologia emocional diminua face à gravidez na maioria dos casos (Buckwalter et al., 1999; Evans et al., 2001), a depressão pós-natal tem uma incidência estimada entre $8 \%$ e $27,5 \%$ (Affonso et al., 2000; Berle et al., 2003; Honey et al., 2003; Honjo et al., 2003; Elliot et al., 2004).

\section{Stress parental}

O constructo stress parental refere-se um tipo de stress especificamente relacionado com a parentalidade (Osteberg \& Hagekull, 2000). Os estudos nesta área, em número e complexidade crescentes, sugerem que este tipo de stress pode ter origem numa variedade de factores, incluindo características das crianças, dos progenitores e contextuais (Reitman et al., 2002).

Os primeiros estudos sobre stress parental focaram as consequências de condições extremas como problemas relacionados com a saúde da criança ou dificuldades económicas, mas os desenvolvimentos mais recentes têm conduzido a uma ênfase no stress parental em circunstâncias normais (Deater-Deckard \& Scarr, 1996). Embora, como referem Deater-Deckard e Scarr (1996), os pequenos stresses do dia-a-dia sejam parte do 'trabalho' de se ser pai ou mãe, o seu efeito negativo 
pode acumular-se e influenciar a qualidade do funcionamento familiar e da relação entre os progenitores e a criança. Maiores níveis de stress parental têm sido associados a vinculação insegura nos filhos, autoritarismo, negligência e abuso parental, qualidade conjugal negativa e depressão materna (Deater-Deckard \& Scarr, 1996; Osteberg \& Hagekull, 2000). Tem sido documentado também que os níveis médios de stress parental são maiores em amostras clínicas do que em amostras não-clínicas (Osteberg \& Hagekull, 2000).

Grande parte da investigação nesta área focou a experiência materna com base na assumpção válida de que as mães, em comparação com os pais, são tipicamente mais sobrecarregadas com as tarefas parentais. Os estudos que incluíram os pais têm produzido evidências mistas quanto à diferença entre os dois sexos. Alguns autores reportaram níveis equivalentes de stress parental nos dois progenitores (Deater-Deckard \& Scarr, 1996), enquanto outros observaram níveis mais elevados nas mulheres (Delmore-Ko et al., 2000). Num estudo com uma amostra não-clínica, Deater-Deckard \& Scarr (1996) verificaram que os níveis de stress parental quer maternos quer paternos se correlacionavam negativamente com a qualidade da relação conjugal e com o nível de participação paterna nas tarefas. Também no estudo de Levy-Shiff (1999), os homens mais envolvidos nos cuidados reportaram menos stress parental no fim do primeiro ano pós-parto. Alguns autores, como Delmore-Ko et al. (2000), sublinham que a adaptação à parentalidade pode ser especialmente exigente para os progenitores sem experiência prévia. No entanto, alguns estudos reportaram maior stress nas mães com mais filhos (Lavee et al., 1996). No estudo de Osteberg \& Hagekull (2000), o efeito da paridade, ainda que significativo, era pequeno.

\section{O Parental Stress Inventory}

O Parenting Stress Index da autoria de Abidin (1983), bem como o modelo teórico subjacente, tem sido amplamente aplicado em estudos sobre o stress parental e goza de considerável popularidade (Reitman et al., 2002). Contudo, quer a versão original com 120 itens, que foi adaptada para a população portuguesa por S.V. Santos (1997), quer o Parenting Stress Index - Short Form (versão abreviada com 36 itens; Abidin, 1995), são apenas apropriadas para pais ou mães com crianças com pelo menos 12 meses.

O Parental Stress Inventory (PSI), da autoria de D.J. Terry (1991a; 1991b) mede o nível de stress parental especificamente no período pós-natal, e é apropriado para os progenitores que estejam casados ou a viver maritalmente. A escala foi desenvolvida com base na literatura e inclui itens adoptados de outras medidas e outros criados para focar motivos de stress específicos do período pós-parto. $\mathrm{O}$ 
inventário foi inicialmente testado numa amostra de 123 pais e mães australianos sem filhos prévios, que participaram num estudo longitudinal sobre a transição para a parentalidade. Os participantes são apresentados com uma lista de dificuldades frequentes depois do nascimento de um filho, incluindo áreas directamente relacionadas com o bebé, problemas na partilha de tarefas com o companheiro/a, e mudança nas rotinas pessoais, relação conjugal e outros relacionamentos. Para cada um dos 23 itens, o pai ou a mãe responde se experienciou aquela dificuldade durante as últimas duas semanas e, se sim, com que intensidade. As quatro opções de resposta são cotadas de 1 a 4, desde 'não sentiu' a 'sentiu e foi muito difícil'. As respostas são somadas para calcular uma nota total de stress parental. O alfa de Cronbach para a escala unidimensional foi o,86 para os pais e o,88 para as mães (Touliatos et al., 2001). No estudo da validade da escala, verificou-se que o PSI se correlacionava com várias medidas concorrentes: bem-estar emocional geral $(-0,63)$, estilo de coping focado no problema $(0,42)$ ou na redução da tensão $(0,54)$, e auto-relatos de eficácia em lidar com a transição para a parentalidade (-0,35). As notas dos dois progenitores também se associavam entre si $(0,44)$ e prediziam os níveis de stress parental quatro meses mais tarde (Touliatos et al., 2001).

Segundo o nosso conhecimento, nenhum instrumento que avalie o stress parental no período pós-parto foi validado para a população portuguesa, bem como são inexistentes estudos que tenham focado esta problemática no nosso país. ${ }^{2}$ Tendo em conta isso, e o claro interesse de se considerarem os niveis de stress parental dos dois progenitores em estudos futuros e intervenções preventivas, apresentamos de seguida os resultados da validação do PSI numa amostra portuguesa.

\section{Método}

\section{Desenvolvimento da versão portuguesa}

No desenvolvimento da versão portuguesa do PSI, que designamos Inventário de Stress Parental no Pós-Parto, procurámos mantê-la o mais semelhante possível ao original em inglês. A tradução para o português foi feita separadamente pela investigadora principal e por uma tradutora profissional, e as duas traduções foram depois comparadas e corrigidas nas discrepâncias pontuais. Finalmente, a versão traduzida final e a original foram analisadas por um grupo de Psicólogos (docentes e clínicos) para garantir a adequação da tradução e validade facial dos itens.

2 Numa investigação recente de Oliveira et al. (2005), foi analisada a percepção neonatal e o stress geral de mães portuguesas no pós-parto, mas não o nivel de stress parental especificamente. 


\section{Participantes}

O grupo inicial de participantes é constituído por 58 pais e 61 mães que responderam ao PSI uma única vez no período pós-parto, num total de 119 progenitores que inclui 57 casais. Os critérios de inclusão foram que os participantes estivessem casados ou a viver maritalmente com o outro progenitor há pelo menos seis meses e fossem maiores de 18 anos. Os participantes foram seleccionados maioritariamente durante a gravidez com recurso a colaboradores em contacto com pessoas elegíveis, sobretudo profissionais de serviços de obstetrícia e de preparação para o parto que obtiveram o consentimento informado (mediante apresentação de carta com explicação dos objectivos e procedimento do estudo e pedido do contacto dos participantes, e sua posterior devolução aos investigadores). Após confirmação por telefone da participação, os questionários foram entregues separadamente a cada progenitor e devolvidos por correio ou pessoalmente à investigadora principal no prazo de uma semana. A recolha dos dados decorreu entre Junho de 2004 e Novembro de 2006.

\section{Procedimento}

A consistência interna foi analisada na amostra total, e separadamente nos pais e nas mães. A validade de constructo foi analisada correlacionando as respostas dos dois progenitores, bem como pela comparação dos resultados do PSI com várias variáveis que têm sido associadas ao stress parental em estudos prévios e que foram medidas no mesmo momento em alguns dos pais e das mães da amostra total: nível de sintomatologia depressiva (47 pais e 47 mães), nível de ansiedade-estado ( 46 pais e 47 mães), nível de ajustamento diádico (32 pais e 34 mães) e nível de envolvimento paterno (55 pais e 59 mães).

\section{Outro Material}

Cada participante respondeu a um questionário com informação sócio-demográfica e relativa à história obstétrica, constituído por questões de resposta curta ou alternativa e de auto-preenchimento e destinado à caracterização da amostra. O nivel de sintomatologia depressiva foi medido com o Inventário de Depressão de Beck (BDI; versão original: Beck Depression Inventory, A. Beck, C.Ward, M. Mendelson, J.Mock \& J. Urbaugh, 1961; versão portuguesa: A.Vaz-Serra \& J. Abreu, 1973); o nivel de ansiedade-estado foi medido com a forma $Y$ do Inventário de Ansiedade Estado-Traço (STAI-Y; versão original: State-Trait Anxiety Inventory, C. D. Spielberger, 1983; versão portuguesa: D.R. Silva, 2003); o nível de ajustamento conjugal foi medido com a Escala de Ajustamento Diádico (DAS; versão original: 
Dyadic Adjustment Scale, G.B. Spanier, 1976; versão portuguesa: Gomez \& Leal, 2008); e o nível de envolvimento paterno no pós-parto foi avaliado pelas respostas maternas à Escala de Confirmação das Expectativas de Suporte Maternas (ECS: versão original: Expectancy Confirmation Scale, M.J. Levitt, S. Coffman, N. Guacci-Franco \& S.C. Loveless, 1993, 1994; versão portuguesa: Gomez \& Leal, 2007).

\section{Resultados}

\section{Características dos participantes}

$\mathrm{Na}$ amostra inicial, a idade média das mulheres era 30 anos $( \pm 3,7$; intervalo: 22-41 anos) e dos homens era 32 anos ( $\pm 4,3$; intervalo: 23-44 anos). A maioria das mulheres $(79 \%)$ e dos homens $(52 \%)$ possuiam a licenciatura (dos restantes, $18 \%$ das mulheres e $34 \%$ dos homens possuíam o $12 .^{\circ}$ ano de escolaridade, e $3 \%$ das mulheres e $14 \%$ dos homens apenas tinham completado o $9 .^{\circ}$ ano). Maioritariamente, os participantes não tinham filhos prévios ( $84 \%$ das mães e $83 \%$ dos pais) e estavam casados ( $77 \%$ das mães e $74 \%$ dos pais; os restantes viviam em união de facto). A gravidez tinha sido planeada em $77 \%$ dos casos. A avaliação decorreu em média 104 dias depois do nascimento $( \pm 45,3$; intervalo: 10-195 dias).

\section{Consistência interna}

Os resultados da análise da consistência interna são apresentados no Quadro 1. Verifica-se que o item 2 ('cólicas do bebé') não cumpre o critério para a aceitação dos itens [correlação com o total corrigido - i.e., o total sem o item - menor que o,2, de acordo Streiner \& Normam (1989)] e que tem um efeito negativo sobre o valor do alfa, o que levou a eliminá-lo da versão final. Em nosso entender, estes valores provavelmente reflectem o facto de as cólicas do recém-nascido serem uma ocorrência normal e passageira do período pós-parto inicial, com que os progenitores aprendem a lidar com relativa facilidade. A versão final, com 22 itens, tem uma boa consistência interna quer considerando a amostra total $(\alpha=0,868)$, quer os grupos de pais $(\alpha=0,898)$ e de mães $(\alpha=0,828)$ separadamente. 
Quadro 1. Consistência interna dos itens na amostra total ( $N=119)$

\begin{tabular}{|c|c|c|}
\hline ITENS & $\begin{array}{l}R \text { item-total } \\
\text { corrigido }\end{array}$ & $\begin{array}{l}\alpha \text { de Cronbach } \\
\text { sem o item }\end{array}$ \\
\hline Problemas na alimentação do bebé &, 386 & ,858 \\
\hline Cólicas do bebé & ,113 & ,868 \\
\hline Lentidão do bebé a estabelecer uma rotina & 394 & ,858 \\
\hline Lentidão do bebé a estabelecer um padrão de sono & ,401 &, 858 \\
\hline Bebé desassossegado &, 356 & ,859 \\
\hline Falta de tempo de sono & 410 & ,857 \\
\hline $\begin{array}{l}\text { Incerteza sobre se a saúde e os progressos do bebé } \\
\text { estão dentro dos limites normais }\end{array}$ & ,349 & ,859 \\
\hline $\begin{array}{l}\text { Ser interrompido/a pelo bebé a meio de estar a fazer } \\
\text { algo }\end{array}$ & 443 & ,857 \\
\hline $\begin{array}{l}\text { Não ser capaz de gerir e coordenar a sua rotina diária } \\
\text { em casa. }\end{array}$ &, 524 & 853 \\
\hline Receber conselhos contraditórios de outras pessoas & ,243 & 863 \\
\hline $\begin{array}{l}\text { Menos tempo com adultos (que não o seu } \\
\text { companheiro/a) }\end{array}$ &, 521 &, 854 \\
\hline Falta de amigos com bebés ou crianças pequenas & 312 & ,860 \\
\hline Menos tempo para fazer as coisas de que gosta &, 510 & ,854 \\
\hline Menos liberdade para fazer o que quiser quando quiser &, 535 & ,854 \\
\hline Falta de estimulação intelectual & ,297 & ,861 \\
\hline Mudanças nos seus compromissos profissionais & ,437 &, 856 \\
\hline Perda de independência &, 502 & ,854 \\
\hline Mudanças na sua relação conjugal & ,658 & 849 \\
\hline Sentir-se mais distante do seu companheiro/a &, 548 &, 852 \\
\hline Passar menos tempo com o seu companheiro/a &, 523 & 853 \\
\hline $\begin{array}{l}\text { Mudanças na relação sexual com o seu } \\
\text { companheiro/a }\end{array}$ &, 535 & 853 \\
\hline $\begin{array}{l}\text { Problemas na partilha das tarefas de cuidado do bebé } \\
\text { com o seu companheiro/a }\end{array}$ & , 472 & 855 \\
\hline $\begin{array}{l}\text { Problemas na partilha das tarefas domésticas com o } \\
\text { seu companheiro/a }\end{array}$ &, 539 &, 853 \\
\hline \multicolumn{2}{|c|}{$\alpha$ de Cronbach do total dos itens (antes da eliminação do item 2) } & ,862 \\
\hline
\end{tabular}

\section{Validade de constructo}

O Quadro 2 apresenta as estatísticas descritivas na amostra total e nas amostras parciais de pais e mães. Nos dois sexos, as notas têm uma amplitude grande e estão bem centradas. As mulheres reportaram níveis de stress ligeiramente mais elevados do que os homens, mas a diferença entre os dois grupos não é significativa ( $t$ de Student $=1,431 ; p=0,155$ ). Na amostra de 57 casais em que os dois progenitores foram avaliados, a correlação entre os valores maternos e paternos ( $r$ de Pearson 
$=, 481 ; p=0,000$ ) é significativa e equivalente à encontrada por Terry (1991a). Finalmente, no Quadro 3 apresentam-se os valores da correlação entre as notas no PSI e nas outras medidas, separadamente para cada sexo. Todas as correlações são muito significativas e conforme o esperado de acordo com a literatura: nos dois progenitores, o stress parental associa-se positivamente ao ajustamento diádico e ao nível de envolvimento paterno depois do nascimento e negativamente ao nível de depressão e de ansiedade.

Quadro 2. Estatísticas descritivas das notas no PSI

\begin{tabular}{cccccc}
\hline & N & Média & d.p. & Mínimo & Máximo \\
\hline Mães & 61 & 41,57 & 8,2 & 25 & 60 \\
Pais & 58 & 39,22 & 9,7 & 26 & 77 \\
Total & 119 & 40,43 & 8,9 & 25 & 77 \\
\hline
\end{tabular}

Quadro 3. Correlação entre as notas no PSI e as outras medidas (testes de Pearson)

\begin{tabular}{ccccc}
\hline & DAS & BDI & STAI-Y & ECS \\
\hline \multirow{3}{*}{ Mães } & $r=-, 499$ & $r=, 462$ & $r=, 626$ & $r=-, 372$ \\
& $p=, 003$ & $p=, 001$ & $p=, 000$ & $p=, 004$ \\
& $(n=34)$ & $(n=47)$ & $(n=47)$ & $(n=59)$ \\
Pais & $r=-, 760$ & $r=, 527$ & $r=, 433$ & $r=-, 409$ \\
& $p=, 000$ & $p=, 000$ & $p=, 003$ & $p=, 002$ \\
& $(n=32)$ & $(n=47)$ & $(n=46)$ & $(n=55)$ \\
\hline
\end{tabular}

\section{Discussão e conclusão}

A versão portuguesa do PSI (ou Inventário de Stress Parental no Pós-parto) apresentou um bom comportamento psicométrico. A consistência interna da versão final de 22 itens é elevada e, à semelhança do que tem sido reportado em estudos prévios, as notas dos dois progenitores correlacionam-se entre si e com medidas de ansiedade, depressão, ajustamento diádico e envolvimento paterno depois do nascimento. Estes resultados indicam que o PSI português pode ser considerado uma medida fidedigna e válida para avaliar o stress parental no pós-parto.

Alguns autores documentaram maiores niveis de stress parental nas mulheres do que nos homens, mas na nossa amostra, bem como no estudo de Deater-Deckard \& Scarr (1996), não houve diferenças entre sexos. É provável, como foi eventualmente o caso na nossa amostra de conveniência, que o sexo não seja uma variável relevante quando os níveis de envolvimento paterno são elevados, já que estes também se associam nos dois sexos à qualidade conjugal (Bonney e col., 1999; Sabattini \& Leaper, 2004) e à satisfação parental (Beitel et al., 1998). 
Os nossos resultados a poiam esta assumpção, já que mostram que nos dois sexos o stress parental se correlaciona negativamente com o ajustamento conjugal e o envolvimento paterno.

Genericamente, notas mais altas no PSI indicam maiores dificuldades na adaptação pós-natal, mas não há um critério relativamente ao qual os valores possam ser comparados para estabelecer um ponto de corte e definir niveis de stress 'normais' ou não. No presente, um nível de stress parental elevado pode definir-se apenas no sentido estatístico, em termos do grau em que um pai ou uma mãe em particular se desvia da média de um grupo de progenitores.

Uma eventual limitação deste estudo é que a amostra não continha uma variedade muito grande de pais e mães. Estudos futuros que incluam outros grupos (por exemplo, grupos clínicos e amostras mais representativas de pais e mães com filhos prévios) podem contribuir para estabelecer melhor a validade da versão portuguesa do PSI, bem como para compreender melhor os factores que se associam ao stress parental no período pós-parto.

Apesar de não ser possivel estabelecer relações causais, os resultados deste e de estudos anteriores claramente indicam que o stress parental é uma variável crítica a ter em conta nas intervenções que foquem o ajustamento dos progenitores e o desenvolvimento da criança. Programas preventivos (ainda antes do nascimento) que ajudem os pais a antecipar diferentes factores de stress, bem como intervenções familiares que ajudem a lidar de forma adequada com as dificuldades, deverão favorecer o bem-estar individual e o desenvolvimento da criança.

\section{Referências}

Abidin, R. R. (1983). Parenting Stress Index - Manual. Charlotsville, VA: Pediatric Psychology Press. Abidin, R.R. (1995). Parenting Stress Index (3rd ed.). Odessa FL: Psychological Assessment Resources.

Affonso, D., De, A., Horowitz, J., and Mayberry, L. (2000). An international study exploring levels of postpartum depressive symptomatology. Journal of Psychosomatic Research, 49, 207-216. Areias, M. E. G., Kumar, R., Barros, H., \& Figueiredo, E. (1996). Correlates of postnatal depression in mothers and fathers. British Journal of Psychiatry, 169, 36-41.

Ausloos, G. (1996). A competência das famílias: Tempo, caos e processo. Lisboa: Climepsi.

Beck, A.T., Ward, C. H., Mendelson, M., Mock, J., \& Erbaugh, J. (1961) An inventory for measuring depression. Archives of General Psychiatry, 4, 561-571.

Beitel, A. H. \& Parke, R. D. (1998). Paternal involvement in infancy: The role of maternal and paternal attitudes. Journal of Family Psychology, 12(2), 268-288.

Berle, J., Aarre, T., Mykletun, D., \& Holsten, F. (2003). Screening for postnatal depression: Validation of the Norwegian version of the Edinburgh Postnatal Depression Scale, and assessment of risk factors for postnatal depression. Journal of Affective Disorders, 76, 151-156. 
Bonney, J.F., Kelley, M.L., \& Levant, R.F.(1999). A model of father's behavioral involvement in child-care in dual-earner families. Journal of Family Psychology, 13, 401-415.

Buckwalter, J., Stanczyk, F., McCleary, C., Bluestein, B., Buckwalter, D., Rankin, K., Chang, L. \& Goodwin, T. (1999). Pregancy, the postpartum and steroid hormones: effects on cognition and mood. Psychoneuroendocrinoloy, 24, 69-84.

Coffman, S., Levitt, M.J., Deets, C. \& Quigley, K.L. (1991). Close relationships in mothers of distressd and normal newborns: Support, expectancy confirmation and maternal well-being. Journal of Family Psychology, 5(1), 93-107.

Colman, L.L. \& Colman, A.D. (1991). Pregnancy - The Psychological Experience. Revised and expanded edition. The Noonday Press: New York.

Deater-Deckard, K. \& Scarr, S. (1996). Parenting stress among dual-earner mothers and fathers: Are there gender differences? Journal of Family Psychology, 10(1), 45-59.

Delmore-Ko, P., Pancer, S., Hunsberger, B. \& Pratt, M. (2000). Becoming a parent: The relation between prenatal expectations and postnatal experience. Journal of Family Psychology, 14(4), 625-640.

Deutsch, F, Servis, L e Payne, J (2001). Paternal participation in child care and its effects on children's self-esteem and attitudes toward gendered roles. Journal of Family Issues, 22(8), 1000-24.

Dienhart, A. (2001). Make room for daddy: The pragmatic potentials of a tag-team structure for sharing paranting. Journal of Family Issues, 22(8), 973-999.

Dulude, D., Bélanger, C., Wright, J. \& Sabourin, S. (2002). High-risk pregnancies, psychological distress and dyadic adjustment. Journal of Reproductive and Infant Psychology, 2O(2), 101-123.

Elliott, S., Leverton, T., Sanjack, M., Turner, H., Cowmeadow, P., Hopkins, J. \& Bushnell, D. (2004). Promoting mental health after childbirth: A controlled trial of primary prevention of postnatal depression. British Journal of Clinical Psychology, 39(3), 223-241.

Evans, J., Heron, J., Francomb, H., Oke, S. \& Golding, J. (2001). Cohort study of depressed mood during pregnancy and after childbirth. British Medical Journal, 323, 257-26o.

Gomez, R. \& Leal, I. (2007). Envolvimento paterno no pós-parto: Estudo de Validação da Escala de Confirmação das Expectativas Maternas de Suporte. "Psicologia: Teoria, Investigação e Prática, 12(2)", 305-317.

Gomez, R. \& Leaal, I. (2008). Ajustamento conjugal: versão portuguesa da 'Dyadic Adjustment Scale'. “Análise Psicológica, 4(26)", 625-638.

Goodman, J.H. (2004). Paternal postpartum depression, its relationship to maternal postpartum depression, and implications for family health. Journal of Advanced Nursing, 45(1), 26-35.

Honjo, S., Arai, S., Kaneko, H. et al. (2003). Antenatal depression and maternal-fetal attachment. Psychopathology, 36, 304-311.

Honey KL, Bennett P, and Morgan M. (2003). Predicting Postnatal Depression. Journal of Affective Disorders, 76, 201-10.

Lavee, Y., Sharlin, S. \& Katz, R. (1996). The effect of parenting stress on marital quality: An integrated mother-father model. Journal of Family Issues, 17, 114-135.

Lazarus, R. S. (1993). Why We Should Think of Stress as a Sugset of Emotion. In L. Goldberg and S. Bredmitz (Eds), Handbook of Stress, Theoritical and Clinical Aspects (pp. 21-39). New York: Free Press. 
Levitt, M.J., Coffman, S., Guacci-Franco, N. \& Loveless, S.C. (1993). Social support and relationship change after childbirth: An expectancy model. Health Care for Women International, 14, 502-512.

Levitt, M.J., Coffman, S., Guacci-Franco, N. \& Loveless, S.C. (1994). Attachment relationships and life transitions. In M.R. Sperling \& W.H. Berman (Eds.), Attachment in adults: Clinical and developmental perspectives (pp.232-255). New York: Guilford.

Levy-Shiff, R. (1994). Individual and contextual correlates of marital change across the transition to parenthood. Developmental Psychology, 30(4), 591-601.

Levy-Shiff, R. (1999). Fathers' cognitive appraisals, coping strategies and support resources as correlates of adjustment to parenthood. Journal of Family Psychology, 13(4), 554-567.

Matthey, S., Barnett, B., Ungerer, J. \& Waters, B. (2000). Paternal and maternal depressed mood during the transition to parenthood. Journal of Affective Disorders, 60(2), 75-85.

Matthey, S., Barnett, B., Howie, P. \& Kavanagh, D. (2003). Diagnosing postpartum depression in mothers and fathers: whatever happened to anxiety? Journal of Affective Disorders, 74, 139-147.

Oliveira, C., Pedrosa, A.A, Monteiro, S., Moura-Ramos M. \& Canavarro, M.C. (2005). Percepção do impacto do nascimento de um filho, tarefas desenvolvimentais e adaptação na transição para a maternidade. IberPsicologia, 10, 2-4.

Osteberg, M. \& Hagekull, B. (2000). A structural modeling approach to the understanding of parenting stress. Journal of Clinical Child Psychology, 29(4), 615-625.

Reitman, D., Currier, R.O. \& Stickle, R.T. (2002). A critical evaluation of the Parenting Stress Index-Short Form (PSI-SF) in a Head Start Population. Journal of Clinical Child and Adolescent Psychology, 31(3), 384-392.

Relvas, A.P. e Lourenço, M.C. (2001). Uma abordagem familiar da gravidez e da maternidade: perspectiva sistémica. In M.C. Canavarro (Ed.), Psicologia da Gravidez e da Maternidade (pp. 105-132). Coimbra: Quarteto Editora.

Sabattini, L. \& Leaper, C. (2004). The relation between mothers' and fathers' parenting styles and their division of labor in the home: Young adults' retrospective reports. Sex Roles, 50 (3/4), 217-225.

Santos, S. V. (1997). Versão portuguesa do Parenting Stress Index (PSI): Validação preliminar. In M. Gonçalves, I. Ribeiro, S. Araújo, C. Machado, L. Almeida, \& M. Simões (Eds.), Avaliação Psicológica: Formas e Contextos (Vol. 5, pp. 139-149). Braga: Associação dos Psicólogos Portugueses.

Silva, D.R. (2003). O Inventário de Estado-Traço de Ansiedade (S.T.A.I.). In Gonçalves, M.M., Simões, M.R., Almeida, L.S. e Machado, C. (Eds), Avaliação Psicológica - Instrumentos validados para a população portuguesa Vol. 1 (pp. 45-63). Coimbra: Quarteto.

Spanier, G.B. (1976). Measuring dyadic adjustment: New scales for assessing the quality of marriage and similar dyads. Journal of Marriage and the Family, 38, 15-28.

Spielberger, C. D. (1983). Manual for the State-Trait Anxiety Inventory (STAI). PaloAlto, CA Consulting Psychologists Press.

Streiner, D.L. e Normam, G.R. (1989). Health Measurement Scales: A practical guide to their development and use. Oxford: Oxford Medical Publications.

Terry, D.J. (1991a). Predictors of levels of subjective stress in a sample of new parents. Australian Journal of Psychology, 43, 29-36.

Terry, D.J. (1991b). Stress, coping and adaptation to new parenthood. Journal of Social and Personal Relationships, 8, 527-547. 
Touliatos, J., Perimutter, B.F. e Straus, M.A. (2001). Handbook of family measurements techniques Vol. 2. Thousand Oaks, Calif.: Sage Publications.

Vaz-Serra, A. \& Abreu, J. (1973). Aferição dos quadros clínicos depressivos I.- Ensaio de aplicação do Inventário Depressivo de Beck a uma amostra portuguesa de doentes deprimidos. Coimbra Médica, 20(6), 623-644.

\section{Parental stress in the postpartum period: Portuguese version of the Parental Stress Inventory}

The Parental Stress Inventory (PSI; D.J. Terry, 1991) was adapted for use with Portuguese parents. In general, the results indicate that the Portuguese version of the PSI is a reliable and valid instrument to measure parental stress in the post-partum period. The Portuguese PSI has a high level of internal consistency $(\alpha=0,862)$. It was further observed that maternal and paternal scores correlate positively to each other and to measures of depression and anxiety, and negatively to measures of marital adjustment and paternal involvement.

KEYWORDS: Parental Stress Inventory; Parenthood; Pregnancy; Post-partum; Family. 